\title{
Simulation and Optimization of Contactless Power Transfer System for Rotary Ultrasonic Machining
}

\author{
Xinwei Wang, Aimin Wang and Xiaolong Wang \\ Beijing Institute of Technology, School of Mechanical Engineering, 5 South Zhongguancun Street, Haidian District, Beijing, China
}

\begin{abstract}
In today's rotary ultrasonic machining (RUM), the power transfer system is based on a contactless power system (rotary transformer) rather than the slip ring that cannot cope with high-speed rotary of the tool. The efficiency of the rotary transformer is vital to the whole rotary ultrasonic machine. This paper focused on simulation of the rotary transformer and enhancing the efficiency of the rotary transformer by optimizing three main factors that influence its efficiency, including the gap between the two ferrite cores, the ratio of length and width of the ferrite core and the thickness of ferrite. The finite element model of rotary transformer was built on Maxwell platform. Simulation and optimization work was based on the finite element model. The optimization results compared with the initial simulation result showed an approximate $18 \%$ enhancement in terms of efficiency, from $77.69 \%$ to $95.2 \%$.
\end{abstract}

\section{Introduction}

Rotary ultrasonic machining (RUM) is a non-traditional machining process that combines the material removal mechanisms of diamond grinding and ultrasonic machining. It comprises of a tool mounted on a rotary spindle that is attached to a piezoelectric transducer to produce the rotary and ultrasonic motion [1]. In RUM machines, the ultrasonic vibration power provided by the ultrasonic generator need to be transferred to the machine tool that rotating in a high speed. The conventional energy transfer method for RUM is via slip ring, which exists a lot of drawbacks and cannot be applied to a spindle rotating in high speed, because it would affect the machining accuracy and working efficiency of RUM [2].

Loosely coupled inductively power transfer (LCIPT) is a novel energy transfer technology that could achieve power transfer without physical contact. LCIPT owns many merits, such as reliability and minimum maintenance requirement. It is unaffected by dirt, dust, water or other chemicals and capable of working in highspeed rotation condition. LCIPT is widely used in areas, such as aerospace [3], medical electronics [4] and electric vehicle [5]. These all proved that LCIPT technology is perfect for rotary transformer. Therefore a contactless power transfer system, also named rotary transformer, using LCIPT technology was designed [6].

Though rotary transformer have been designed for years and used in many areas, optimization research and articles related to rotary transformer for URM are relatively scarce. This paper focused on optimization of the rotary transformer, by optimizing the gap between the two ferrite cores, the ratio of length and width of the ferrite core and the thickness of ferrite, to enhance its efficiency. The optimization work of rotary transformer was performed and validated through Maxwell platform based on a finite element model of rotary transformer of URM that had already been built in former work [7].

\section{Principle of rotary transformer}

Based on the principle of electromagnetic mutual inductance theory, the rotary transformer contains a set of pot ferrite cores with windings fitted into both of them (Fig. 1). The primary pot core is static, and the winding in it is connected to the ultrasonic generator, which produces electrical power of ultrasonic frequency. The secondary pot core is fixed to the rotary spindle (spinning when working) and connected to the ultrasonic transfer, and winding in it is induced to generate an alternating current of the same frequency as the primary winding to achieve the power transfer.

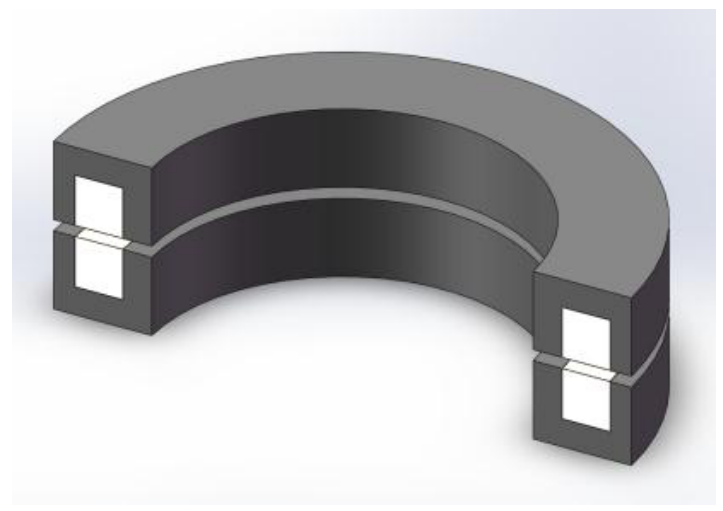

Figure 1. Contactless power transfer system for RUM 


\section{Simulation of rotary transformer}

The simulation of the rotary transformer was carried out on the Maxwell platform. The transient magnetic analytical module of Maxwell was adopted to simulate the mutual inductance coupling model of rotary transformer. Firstly, the mechanical model of the RUM rotary transformer has been built with dimensions according to the (Fig. 2) and Table 1, and the core material was set to ferrite with a relative magnetic permeability of 2300 . Then the mesh operations, primary and secondary windings, the boundary conditions and the later processing of the contours of the electric and magnetic field have been set. The ultrasonic-frequency $(20 \mathrm{KHz}) \mathrm{AC}$ signal is assigned to the primary winding. It has been proved the spinning movement of the secondary ferrite core has little influence of the efficiency of the rotary transformer [6], so it is neglected in the simulation work.

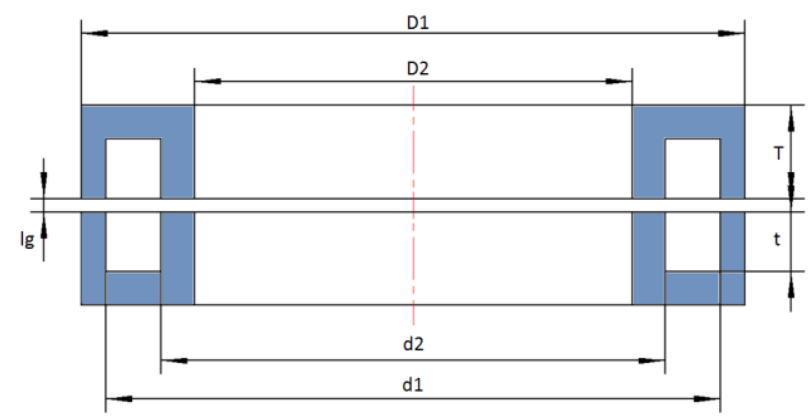

Figure 2. The dimensions of rotary transformer

Table 1. The initial dimensions of rotary transformer before optimization

\begin{tabular}{|c|c|}
\hline$D_{1} / \mathrm{mm}$ & 100 \\
\hline$D_{2} / \mathrm{mm}$ & 66 \\
\hline$d_{1} / \mathrm{mm}$ & 92.6 \\
\hline$d_{2} / \mathrm{mm}$ & 76 \\
\hline$T / \mathrm{mm}$ & 14 \\
\hline$t / \mathrm{mm}$ & 9 \\
\hline$l g / \mathrm{mm}$ & 1 \\
\hline
\end{tabular}

The simulation results were showed in (Fig. 3) showed the relationship between the secondary winding (winding2) and primary winding (winding1). The simulation results of the rotary transformer were same with the theory analysis. The frequency of the secondary winding is the same as the primary winding, but the phase of the secondary winding is half cycle behind the primary winding, due to the principle of electromagnetic mutual inductance. The amplitude inducted current (secondary winding) $I_{2}$ is smaller than the input current (primary winding) $I_{1}$ because of the energy loss during the transfer process.

For a better understanding of the simulation results of the rotary transformer, the current density distribution of rotary transformer at $15 \mu \mathrm{s}$ was showed in (Fig. 4). The result is corresponding with (Fig. 3) that the current density of the primary winding is higher than that of the secondary winding.

The magnetic field $B$ of rotary transformer at $25 \mu \mathrm{s}$ was showed in (Fig. 5). We can see that the distribution of magnetic fields $\mathrm{B}$ is relatively equal in the ferrite core at about $300 \mathrm{mT}$, which is the perfect working area for ferrite.

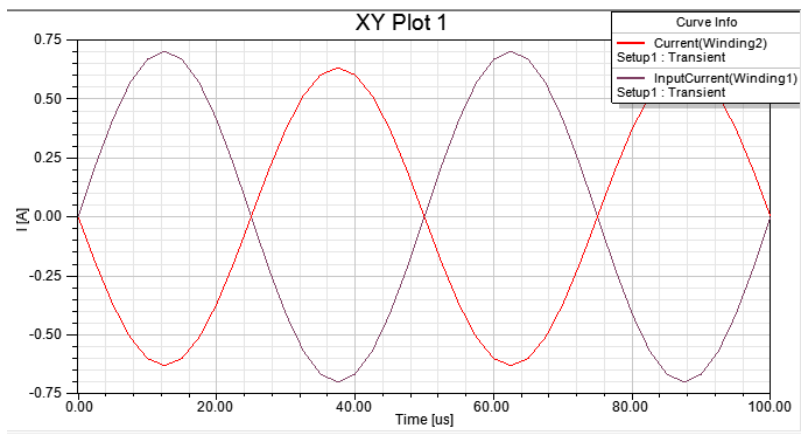

Figure 3. Simulation results for Winding2 under the ImputCurrent Winding1

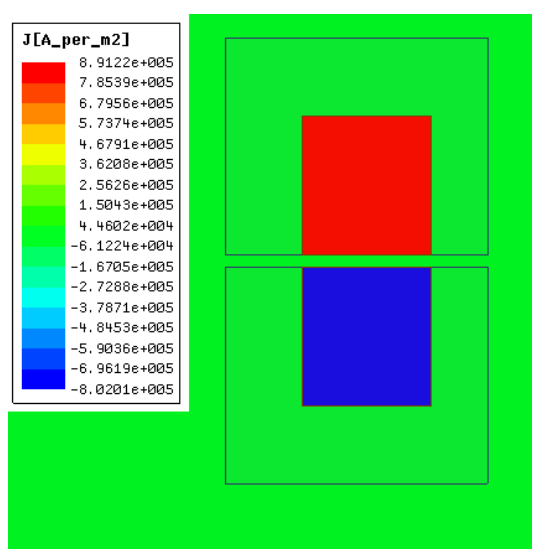

Figure 4. Current density distribution of rotary transformer at $15 \mu \mathrm{s}$

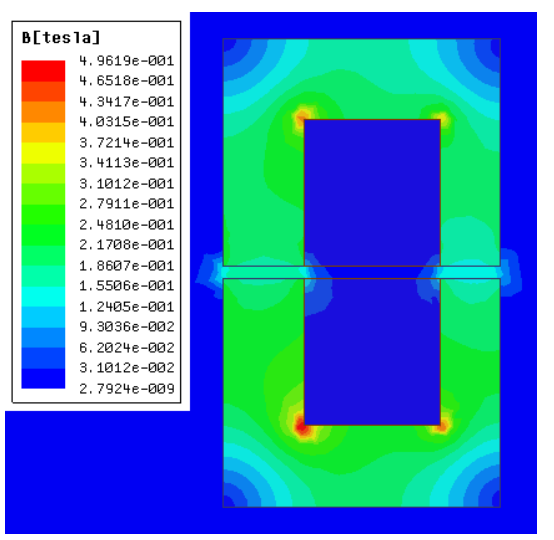

Figure 5. Magnetic field B of rotary transformer at $25 \mu \mathrm{s}$

\section{Optimization of rotary transformer}

Based on the simulation results, the optimization work begins. The main parameters of the rotary transformer, including $D_{1}, D_{2}, d_{1}, d_{2}, T, t, l g$ were all considered in the optimization work. The optimization work mainly considered three factors. First the gap between the two 
ferrite cores, then the ratio of length and width of the ferrite core and finally the thickness of ferrite. The initial dimensions of the rotary transformer are showed in (Fig. 2 ) and Table. 1. The transfer efficiency is defined as $E$,

$$
E=\frac{I_{2}^{2} Z_{2}}{I_{1}^{2} Z_{1}}
$$

where $Z_{1}$ and $Z_{2}$ is the impedance of the primary ferrite core and secondary ferrite core, and since they are identical $Z_{1}=Z_{2}$.

\subsection{Gap between two ferrite cores}

The change of the gap between two ferrite cores $l g$ from $0.5 \mathrm{~mm}$ to $3 \mathrm{~mm}$ has been showed in (Fig. 6). And the relationship between $l g$ and transfer efficiency $E$ is showed in (Fig. 7). With all the other parameters unchanged, but the air gap between the two ferrite cores lg changing from $0.5 \mathrm{~mm}$ to $3 \mathrm{~mm}$, the efficiency of the rotary transformer reduced from approximately $86 \%$ to $55 \%$. From this point of view, the larger the air gap, the lower the transfer efficiency.

This could be explained by the bigger the air gap the more amount of energy will loss to the air, so the less the transfer efficiency. However, due to the physical and machining restrictions, the air gap couldn't be too small. Therefore $0.8 \mathrm{~mm}$ was chose for the air gap of the rotary transformer, and the latter optimization work of the ratio of the length and width of the rotary transformer and the thickness of ferrite were based on this air gap.

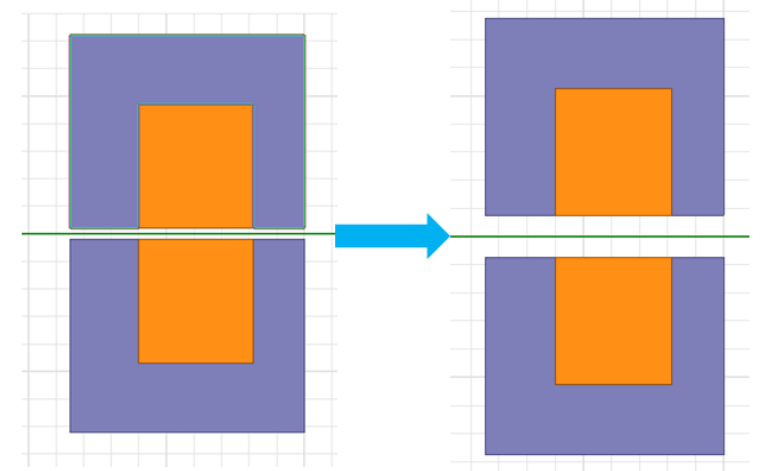

Figure 6. Optimization of the gap between two ferrite cores

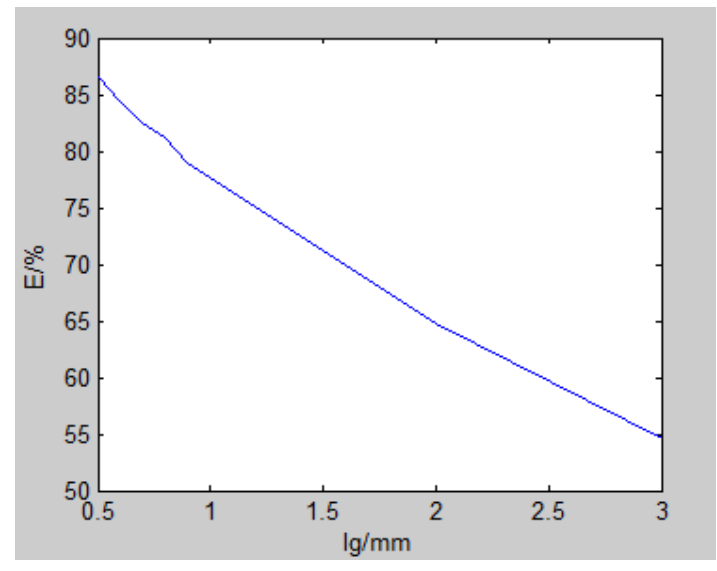

Figure 7. The relationship between $\lg$ and $E$

\subsection{Ratio of length and width.}

Define the ratio of the length and width as $R$,

$$
R=\frac{D_{1}-D_{2}}{2 T}
$$

Changing $R$ from 1.2 to 2.8 by changing $T$ (Fig. 2) of the rotary transformer while the length of the ferrite core D1, D2, d1, d2 (Fig. 2) was kept unchanged, like showed in (Fig. 8), the rotary transformer become more short.

The relationship between $R$ and efficiency $E$ was showed in (Fig. 9). We can see that with the increasing of $R$ from 1.2 to 2.8 , the efficiency $E$ was also increasing, though with a decreasing acceleration, from $81 \%$ to $95 \%$, respectively.

Due to this fact, it is better to design the rotary transformer in large length and small width, as long as there is enough space for the winding, and the rotary transformer could be suitable to fit in the spindle of the ultrasonic machining tool.

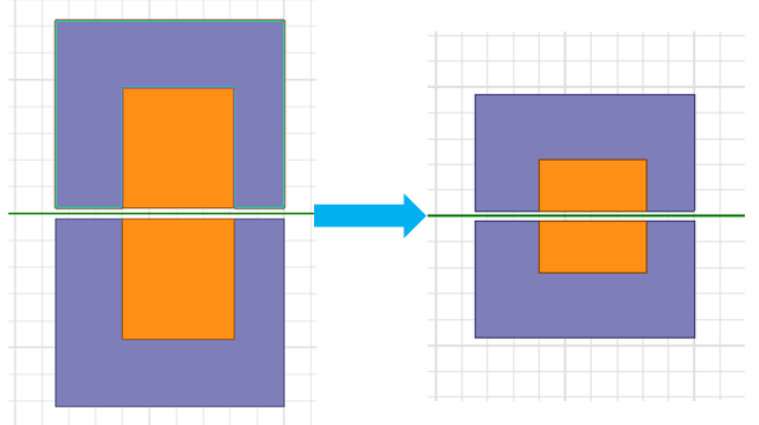

Figure 8. Optimization of ratio of length and width

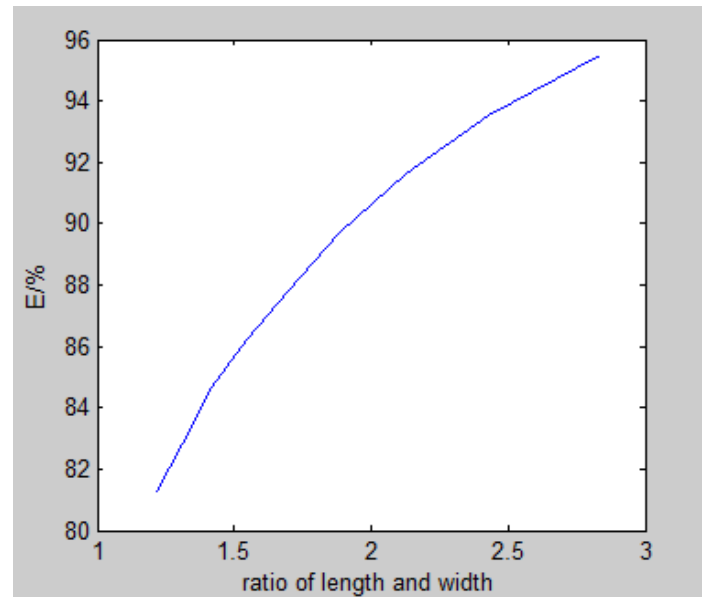

Figure 9. The relationship between ratio of length and width and $E$

\subsection{Thickness of the ferrite cores}

Researches have also showed that the thickness of the ferrite cores could help enhance the efficiency of the rotary transformer. The change of the thickness of the rotary transformer of from $5 \mathrm{~mm}$ to $12 \mathrm{~mm}$ was showed in (Fig. 10). The relationship between the thickness of the 
ferrite core and the transfer efficiency $E$ is showed in (Fig. 11).

With the thickness of ferrite increasing, the efficiency of the rotary transformer also increased, through with a decreasing acceleration. It could be explained by the theory that the thicker the ferrite is, the better it is at accumulating the magnetic power, which means less energy loss.

However, the ferrite of the rotary cannot be too thick for many reasons. First, the magnetic field $B$ distribution is related to the thickness of the ferrite. The thicker the ferrite the smaller the magnetic field $B$, and there is a perfect working area for magnetic field $B$ which is 300 mT [8]. Secondly, the size of the ferrite core should be suitable for it to assembling to the spindle of the ultrasonic machine, so the ferrite core cannot be too big. Finally, the ferrite cannot be fully used if it is too thick, so for the energy efficiency, the ferrite of the rotary should not be too thick.

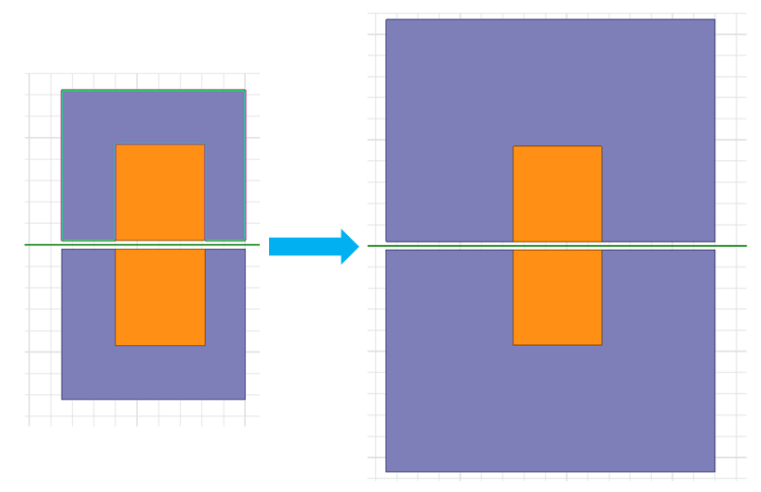

Figure 10. Optimization of the thickness of the ferrite

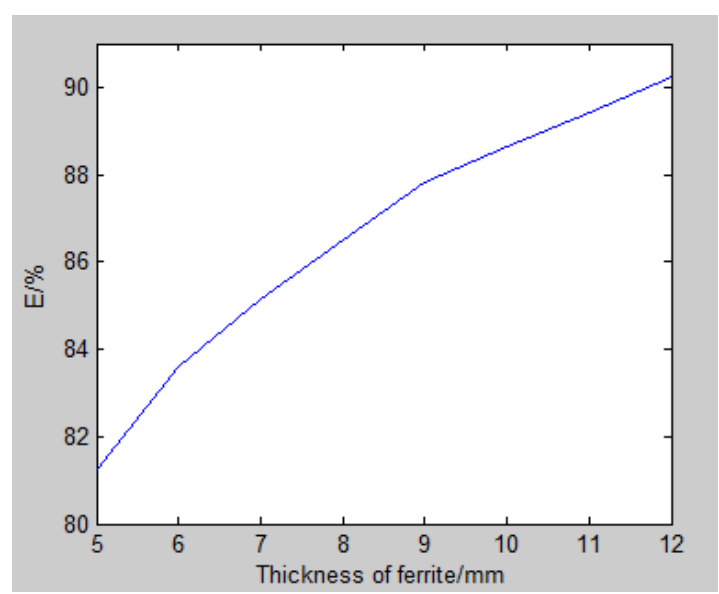

Figure 11. The relationship between thickness of ferrite and $E$

\section{Conclusion}

Based on the simulation result, we conclude the designed rotary transformer can be used to transfer the electromagnetic energy from the stationary primary windings to the rotating secondary windings, so the ultrasonic spindle can obtain stable energy in a high rotating state.

In the optimization part, three main factors that affect the transfer efficiency of the rotary transformer, gap between the two ferrite cores, the ratio of length and width of the ferrite core and the thickness of ferrite that affect the efficiency of the rotary transformer were all analysed on Maxwell platform. We concluded that the less the gap is, the bigger the ratio of length and width is, and the thicker the ferrite, the more efficient the rotary transformer becomes.

However, the size and physical conditions of rotary ultrasonic machine should also be taken into account such that the rotary transformer could be assembled onto the rotary ultrasonic machine. It means that the gap between the two ferrite cores is impossible to be zero (greatest efficiency), the ratio of length and width is impossible to be infinity and that the ferrite could not be too thick in the dimension control of rotary transformer.

Based on all these factors, the optimal parameters of the rotary transformer can be obtained. Suitable gap between the two ferrite cores, the ratio of length and width of the ferrite core and the thickness of ferrite could be chosen. The rotary transformer can greatly improve the transfer efficiency and work in the best state to meet the maximum power transfer principle, reducing the unnecessary electrical and magnetic energy loss, with power transfer efficiency up to $95 \%$. The simulation results showed an excellent result for the rotary transformer optimization, though experiments have not been conducted yet, the experimental efficiency of rotary transformer is expected to be up to $90 \%$.

\section{References}

1. N. Churi, Rotary ultrasonic machining of hard-tomachine materials, B. S., University of Mumbai, India, (2002).

2. L. P. Liu, Study on the key technologies of rotary ultrasonic machining based on accessing of the machine tool, Tianjin Univerosity, (2011).

3. K. D. Papastergiou and D. E. Macpherson, An airborne radar power supply with contactless transfer of energy-part I: Rotating transformer, IEEE Trans. Ind. Electron, 5-45: 2874-2884, (2007).

4. P. T. Theilmann, Wireless power transfer for scaled electronic biomedical implants, University of California, San Diego, (2012).

5. C. P. Choudhury, Modeling and analysis of an inductive power transfer system, Queen's University, December, (1989).

6. Z. L. Long, X. W. Lin, W. Yuan and J. G. Zhang, Modeling on a non-contact power transmission system in ultrasonic machining, Proceeding of the IEEE International Conference on Robotics and Biomimetics, ROBIO, Shenzhen, China, December (2013).

7. X. W. Wang, A. M. Wang, X. L. Wang, Modeling analysis of contactless power transfer system for rotary ultrasonic machining, submitted to Applied mechanics and materials, (2015).

8. H. Hao, FEM Analysis of Contactless Power Transmission System for Ultrasonic Assisted Machining, Dalian University of Technology, (2013). 